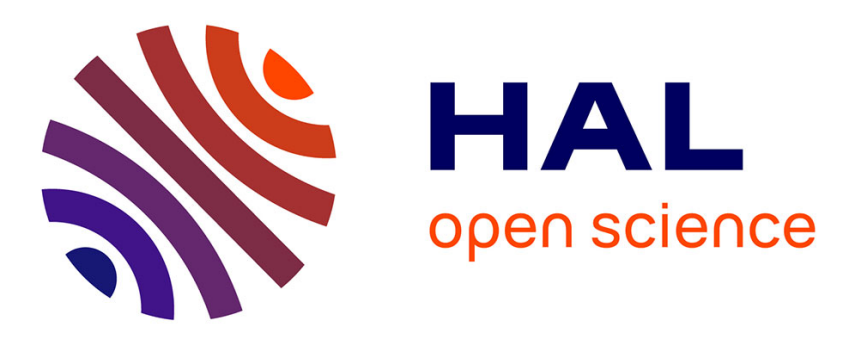

\title{
Usefulness of diffusion derived vessel density computed from a simplified IVIM imaging protocol: an experimental study with rat biliary duct blockage induced liver fibrosis.
}

Gen-Wen Hu, Cun-Jing Zheng, Wei-Xiang Zhong, Dan-Ping Zhuang, Ben-Heng Xiao, Yì Xiáng, J Wáng

\section{To cite this version:}

Gen-Wen Hu, Cun-Jing Zheng, Wei-Xiang Zhong, Dan-Ping Zhuang, Ben-Heng Xiao, et al.. Usefulness of diffusion derived vessel density computed from a simplified IVIM imaging protocol: an experimental study with rat biliary duct blockage induced liver fibrosis.. 2021. hal-03221290

\author{
HAL Id: hal-03221290 \\ https://hal.science/hal-03221290 \\ Preprint submitted on 11 May 2021
}

HAL is a multi-disciplinary open access archive for the deposit and dissemination of scientific research documents, whether they are published or not. The documents may come from teaching and research institutions in France or abroad, or from public or private research centers.
L'archive ouverte pluridisciplinaire HAL, est destinée au dépôt et à la diffusion de documents scientifiques de niveau recherche, publiés ou non, émanant des établissements d'enseignement et de recherche français ou étrangers, des laboratoires publics ou privés. 
Usefulness of diffusion derived vessel density computed from a simplified IVIM imaging protocol: an experimental study with rat biliary duct blockage induced liver fibrosis.

Gen-Wen Hu ${ }^{1 \#}$, Cun-Jing Zheng ${ }^{2 \#}$, Wei-Xiang Zhong ${ }^{3}$, Dan-Ping Zhuang ${ }^{1}$, Ben-Heng Xiao ${ }^{2}$, Yì Xiáng J. Wáng ${ }^{2 *}$.

\#, these two authors contributed equally to this work.

1. Department of Radiology, Shenzhen People's Hospital (The Second Clinical Medical College, Jinan University; The First Affiliated Hospital, Southern University of Science and Technology), Shenzhen, Guangdong Province, China.

2. Department of Imaging and Interventional Radiology, Faculty of Medicine, The Chinese University of Hong Kong, Shatin, New Territories, Hong Kong SAR, China

3. Department of Pathology, The First Affiliated Hospital, Zhejiang University School of Medicine, Hangzhou, Zhejiang Province, China.

*Correspondence to:

Dr. Yì Xiáng Wáng. Department of Imaging and Interventional Radiology, Faculty of Medicine, The Chinese University of Hong Kong, Shatin, New Territories, Hong Kong SAR. Email: yixiang_wang@cuhk.edu.hk 


\begin{abstract}
Aim: Liver vessel density can be evaluated by DDVD (diffusion derived vessel density): $\operatorname{DDVD}(\mathrm{bOb1})=\mathrm{Sb0} / \mathrm{ROIarea0}-\mathrm{Sb} 1 / \mathrm{ROlarea1}$, where Sb0 and Sb1 refer to the liver signal when $b$ is 0 or $1\left(\mathrm{~s} / \mathrm{mm}^{2}\right)$; ROlarea 0 and ROlarea1 refer to the region-of-interest on $b=0$ or 1 images. Sb1 and ROlarea1 may be replaced by Sb25 and ROlarea25 or Sb50 and ROlarea50 (i.e., by images of $b=25$ or $50 \mathrm{~s} / \mathrm{mm}^{2}$ ). With rat biliary duct ligation (BDL) model, this study assessed the usefulness of liver DDVD computed from a simplified IVIM imaging protocol.

Materials and Methods: Male Sprague-Dawley rats were used, and 1, 2, 3, 4 weeks post BDL had rat number of 5, 5, 5,3 respectively. Partial recanalization $(n=12)$ was performed with rats had BDL for 7 days and MRI was done mean 14 days after recanalization. Liver diffusion MR images were acquired using with a $3.0 \mathrm{~T}$ magnet with a $b$-value distribution of $0,30,50,75,100$, $150,300,700,1000 \mathrm{~s} / \mathrm{mm}^{2}$. DDVDmean (control rats $\mathrm{n}=6$ ) was the mean of DDVD(b0b25) and DDVD(b0b50). IVIM fitting started from $b=0$ with segmented fitting and a threshold $b$ of 50 $\mathrm{s} / \mathrm{mm}^{2}$ ( $n=5$ for control rats). Three 3-D space was constructed using PF, $D_{\text {slow, }}$ and $D_{\text {fast }}$ PF, $D_{\text {slow, }}$ and $D D V D_{\text {mean }} ;$ or $P F, D_{\text {fast, }}$ and $D D V D_{\text {mean }}$.

Results: BDL week 1, 2, 3, 4 rats and partial recanalization rats had mean liver fibrosis METAVIR score of 1.5, 2.4, 3.25, 3.7 and 2.42 respectively. The control rats and BDL rats $(n=18)$ had a liver DDVD $_{\text {mean }}$ of $84.0 \pm 26.2$ and $44.7 \pm 14.4$ au/pixel $(p<0.001)$. Each diffusion MRI parameter alone could not totally separate healthy livers and all fibrotic livers ( $n=30, B D L$ and BDL+ recanalization rats). All 3-D spaces totally separated healthy livers and all fibrotic livers. The mean relative distance between healthy liver cluster and fibrotic liver cluster was 0.331 for PF, $D_{\text {slow, }}$ and $D_{\text {fast }} ;$ Dfast, 0.381 for or $P F, D_{\text {fast }}$, and $D D V D_{\text {mean }}$; and 0.384 for $P F, D_{\text {slow, }}$ and $D D V D_{\text {mean }}$. Conclusion: A combination of $\mathrm{PF}, \mathrm{D}_{\text {slow, }}$ and $\mathrm{D}_{\text {fast }}$ allows a total separation of all healthy livers and fibrotic livers, with the integration of DDVD improved the separation.
\end{abstract}

Keywords: Magnetic resonance imaging (MRI); intravoxel incoherent motion (IVIM); diffusion; perfusion; liver; fibrosis; viral hepatitis 
On liver diffusion weighted imaging, blood vessels show high signal when there is no diffusion gradient $\left(b=0 \mathrm{~s} / \mathrm{mm}^{2}\right)$, while show low signal even when very low b-values (such as $b=1 \mathrm{~s} / \mathrm{mm}^{2}$ ) is applied. Recently Wang [1] proposed that liver vessel density can be measured by a diffusion weighted imaging derived surrogate biomarker (DDVD: diffusion derived vessel density):

$\operatorname{DDVD}_{(\mathrm{bob} 1)}=\mathrm{Sb0} / \mathrm{ROIarea0}-\mathrm{Sb} 1 /$ ROlarea1

where Sb0 refers to the measured liver signal intensity when $b=0 \mathrm{~s} / \mathrm{mm}^{2}$, and Sb1 refers to the measured liver signal intensity when $b=1 \mathrm{~s} / \mathrm{mm}^{2}$. ROlarea0 and ROlarea1 refer to the region-of-interest (ROI) on $b=0 \mathrm{~s} / \mathrm{mm}^{2}$ and $b=1 \mathrm{~s} / \mathrm{mm}^{2}$ images, respectively, and '/' means 'divided by'.

Sb1 and ROlarea1 can also be approximated by other low $b$-value diffusion image's data [2, 3].

While we advocate sampling $16 b$-values for liver IVIM imaging [4, 5], simplified IVIM protocols using less $b$-values are often adopted clinically, and very low $b$-value images are not always acquired. With a rat biliary duct ligation model, this study assessed the usefulness of liver DDVD measure computed from a simplified IVIM imaging protocol, alone and in combination with other IVIM parameters, to assess liver fibrosis. Instead of DDVD computed from the signal difference between on $b=0 \mathrm{~s} / \mathrm{mm}^{2}$ and $b=2^{\sim 15} \mathrm{~s} / \mathrm{mm}^{2}$ images as we previously reported [1-3], in this study DDVD was computed from the signal difference between on $b=0 \mathrm{~s} / \mathrm{mm}^{2}$ and $b=30$ or $50 \mathrm{~s} / \mathrm{mm}^{2}$ images.

Material and Methods

Animals:

Male Sprague-Dawley rats (weight: $300 \pm 20 \mathrm{~g}$ ) were used. For bile duct blockage ligation (BDL) surgery, rats' lower segment of common bile duct ligated. Recanalization operation was performed with rats common bile duct isolated and ligated for 7 days. Via a laparotomy, the ligated distal end of the common bile duct was dissected, and an anastomosis was established between the common bile duct and the jejunum, which was sectioned $5 \mathrm{~cm}$ from the duodenojejunal angle [6]. 
$\mathrm{N}=6\left(8^{*}\right)$ rats: control without intervention, $\mathrm{MRI}$ and histology

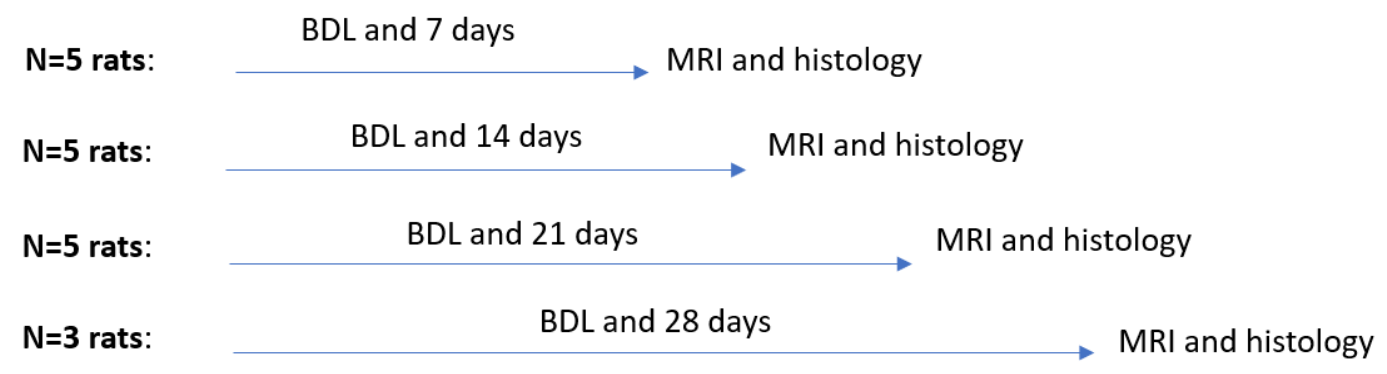

$\mathrm{N}=12$ rats: $\underset{\text { Re-canalization operation }}{\longrightarrow} \mathrm{BDL}$ and 7 days $\mathrm{14}$ days (mean) $\mathrm{MRI}$ and histology

Fig-1. The experimental procedures for rats. In total 8 control rats and 30 rats with surgery were studied. *: Two of the control rats failed the MRI examination.

Magnetic resonance imaging:

MRI images were acquired with a 3.0T magnet (Ingenia, Philips Healthcare, Best, Netherlands) with a 4-channel animal coil (Suzhou Zhongzhi Medical Technologies Co., Suzhou, China). Rats were anesthetized and placed in a prone position. An upper abdominal belt was used to minimize liver motion. For liver diffusion weighted imaging MRI, the parameters were as follows: $\mathrm{TR} / \mathrm{TE}=2000 / 55 \mathrm{~ms} ; \mathrm{EPI}$ factor $=63, \mathrm{FOV}=50 \times 50 \mathrm{~mm}$, slice thickness $=3 \mathrm{~mm}$, number of slices $=9$, matrix $=64 \times 63$, motion probing gradients in three orthogonal axes. SPIR technique (Spectral Pre-saturation with Inversion-Recovery) was used for fat suppression. The $b$-value distribution was $0,30,50,75,100,150,300,700,1000 \mathrm{~s} / \mathrm{mm}^{2}$. NEX was 3 and 4 respectively for images of $b=700$ and $b=1000$, while NEX was 1 for images of other $b$-values.

Image analysis 
All quantitative data analysis was implemented in MATLAB (MathWorks, Natick, MA, USA). For DDVD/area measurement, ROI for liver parenchyma was segmented on the $b=0 \mathrm{~s} / \mathrm{mm}^{2}$ image (resulting in ROI area of area0) and the $b=25$ (or 50) $\mathrm{s} / \mathrm{mm}^{2}$ image (resulting in ROI area of area25 or area50) respectively (Figure-2) [2].

Two parameters were obtained as

$\operatorname{DDVD}_{(\mathrm{bob25})}=\mathrm{SbO} / \mathrm{ROlarea0}-\mathrm{Sb} 2 / \mathrm{ROIarea25}$

$\operatorname{DDVD}_{(\mathrm{b} 0 \mathrm{~b} 50)}=\mathrm{Sb0} / \mathrm{ROlarea0}-\mathrm{Sb} 50 /$ ROlarea50

The average of area weighted DDVDs of included liver axial slice's was the value of the liver. For each rat, measurement was conducted twice, and the mean of the two measurement was adopted as the final result. $D D V D_{\text {mean }}$ is then the mean of DDVD(b0b25) and DDVD(b0b50).

The bi-compartmental IVIM modeling is based on Equation-4:

$\mathrm{SI}_{(\mathrm{b})} / \mathrm{SI}_{(0)}=(1-\mathrm{PF}) \times \exp \left(-b \times \mathrm{D}_{\text {slow }}\right)+\mathrm{PF} \times \exp \left(-b \times \mathrm{D}_{\text {fast }}\right)$

where $\mathrm{SI}_{(\mathrm{b})}$ and $\mathrm{SI}_{(0)}$ denote the signal intensity of images acquired with the $b$-factor value of $b$ and $b=0 \mathrm{~s} / \mathrm{mm}^{2}$, respectively. $D_{\text {slow }}($ or $D)$ is the diffusion coefficient representing the slow 'pure' molecular diffusion (unaffected by perfusion). IVIM analysis follow the principle of our previous studies for human subjects $[1,4,5,7]$. The standard analysis was segmented fitting with a threshold $b$ of $50 \mathrm{~s} / \mathrm{mm}^{2}$ [8]. For each rat, measurement was conducted twice, and the mean of the two measurement was adopted as the final result. 

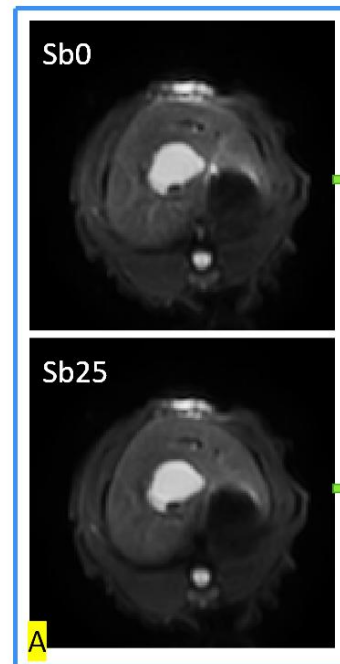

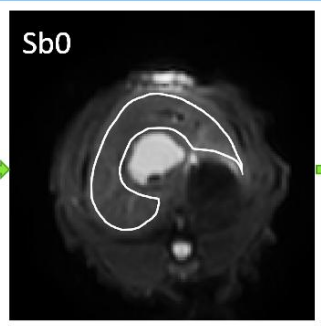

$\mathrm{Sb} 25$

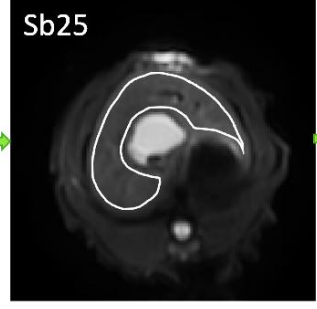

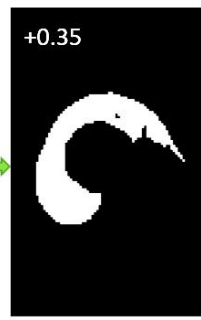

$-0.25$

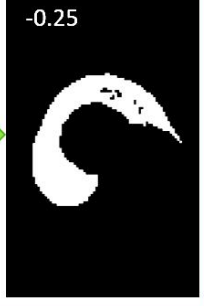

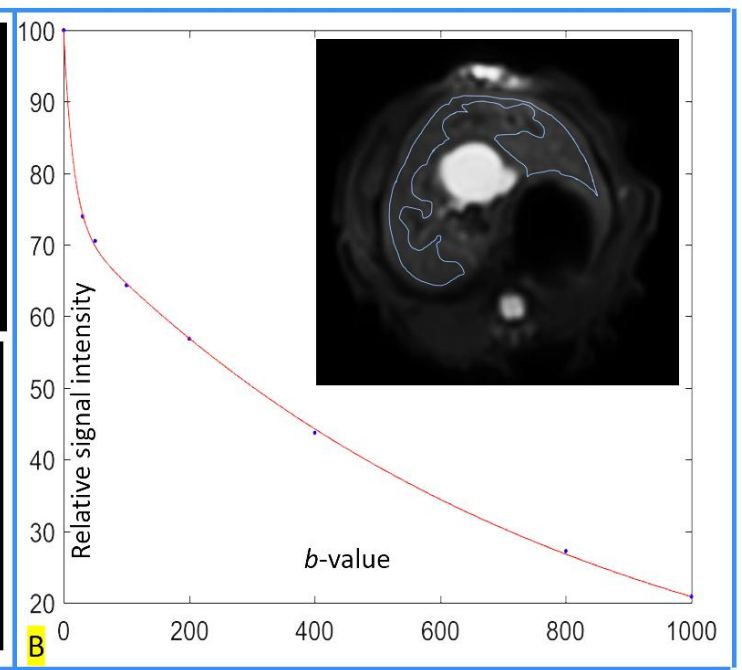

Fig-2. Region of interest (ROI) segmentation of right liver parenchyma for DDVD and IVIM parameter calculation. A, DDVD evaluation. ROI is segmented on the $b=0 \mathrm{~s} / \mathrm{mm} 2$ image and the $b=25 \mathrm{~s} / \mathrm{mm} 2$ image. Then, on the $b=0 \mathrm{~s} / \mathrm{mm} 2$ image, a threshold is selected to remove all pixels of vessels that are of bright signal; on the $b=25 \mathrm{~s} / \mathrm{mm} 2$ image, in addition to the removal of bright signal pixels, a threshold is selected to remove all pixels of vessels that are of "signal void". B, IVIM evaluation. An example of ROI drawn over right liver parenchyma for IVIM bioexponential processing, and the resulting liver signal curve over increasing $b$-values, are shown.

Diffusion parameters were used to build 3-D model to predict the best separation between the non-fibrotic livers and fibrotic livers. The maximized the relative distance between the healthy livers and the fibrotic livers is favored. As previously described [2], to estimate the relative distance between the measures of health livers and fibrotic livers, for each rat's results, the DDVD mean, $P F, D_{\text {slow }}, D_{\text {fast }}$ values were normalized to range between 0 and 1 according to the formula: $\left(x_{i}-x_{\min }\right) /\left(x_{\max }-x_{\min }\right) . x_{\max }$ means the largest value of $\mathrm{DDVD}_{\text {mean }}, \mathrm{PF}, \mathrm{D}_{\text {slow, }}$ or $D_{\text {fast }}$ in this study; and $x_{\text {min }}$ means smallest value of $D D V D_{\text {mean }}, P F, D_{\text {slow }}$, or $D_{\text {fast }}$ in this study. As the measured values differed substantially among these four parameters, this normalization gives each parameter equal weighting. A 3-D coordinate system was initially constructed with $P F, D_{\text {slow }}$ and $D_{\text {fast }}$, and then $D_{\text {fast }}$ or $D_{\text {slow }}$ was replaced with $D D V D_{\text {mean }}$. With support vector machine (SVM) approach, the best separating plane between health livers and fibrotic livers 
was defined as $A^{*} X+B^{*} Y+C^{*} Z+D=0$. In the 3-d coordinate system, the distance between point $\left(X_{0}, Y_{0}, Z_{0}\right)$ and plane $A^{*} X+B^{*} Y+C^{*} Z+D=0$ was calculated according to following equation:

$\frac{\left|A * X_{0}+B * Y_{0}+C * Z_{0}+D\right|}{\sqrt{A^{2}+B^{2}}+C^{2}}$

(5)

The mean distance of points representing healthy livers to the plane and mean distance of points representing fibrotic livers to the line were calculated, and then these two distances were added up.

In total, two control rats failed MRI due to movement during the examination, with motion artifacts and low signal-to-noise ratio. In addition, for one additional control rat, only the DDVD measurement was available, with IVIM measure unusable due to artifact on high $b$-value images. MRI of all surgery rats could be analyzed.

After the MRI, the rats had histological examination of the liver with Hematoxylin-eosin staining as well as picrosirius staining. Histology was assessed independently by a histopathologist, with diagnosis for liver fibrosis was based on the METAVIR criteria. A mean liver fibrosis score for each experimental rat group was assigned by the sum of fibrosis staging value divided by the number of the rat in the group. 
Results

MRI morphologically, all control rats livers had normal appearance, while all rats with BDL had dilated biliary duct system. Of the rats with re-cannulation operation, biliary duct system remained dilated on $\mathrm{MRI}$, with none showing apparent resolvement of biliary duct dilatation. The histopathological grading for liver fibrosis is shown in table-1. For the re-cannulation group, diverse liver fibrosis stages were noted. However, the mean of liver fibrosis score of the recannulation group was higher than the week-1 group (2.42 vs 1.6), suggesting overall progress of liver fibrosis, rather than overall recovery, following the re-cannulation surgery.

Table-1. METAVIR score of the experimental rats livers with surgery intervention.

\begin{tabular}{|c|c|c|c|c|c|c|}
\hline BDL duration & rat number & stage-1 & stage-2 & stage-3 & stage-4 & $\begin{array}{c}\text { mean fibrosis } \\
\text { score }\end{array}$ \\
\hline 7 days & 5 & 2 & 3 & & & 1.6 \\
\hline 14 days & 5 & & 3 & 2 & & 2.4 \\
\hline 21 days* & 4 & & 1 & 1 & 2 & 3.25 \\
\hline 28 days & 3 & & & 1 & 2 & 3.7 \\
\hline Re-cannulation & 12 & 2 & 5 & 3 & 2 & 2.42 \\
\hline
\end{tabular}

* One rat in this group missed histology examination. 
The control rats had a liver DDVD ${ }_{\text {mean }}$ of $84.0 \pm 26.2 \mathrm{au} /$ pixel, which was significantly higher than that of the rats with BDL $(44.7 \pm 14.4 \mathrm{au} /$ pixel, $\mathrm{p}<0.001)$ (Fig-3).
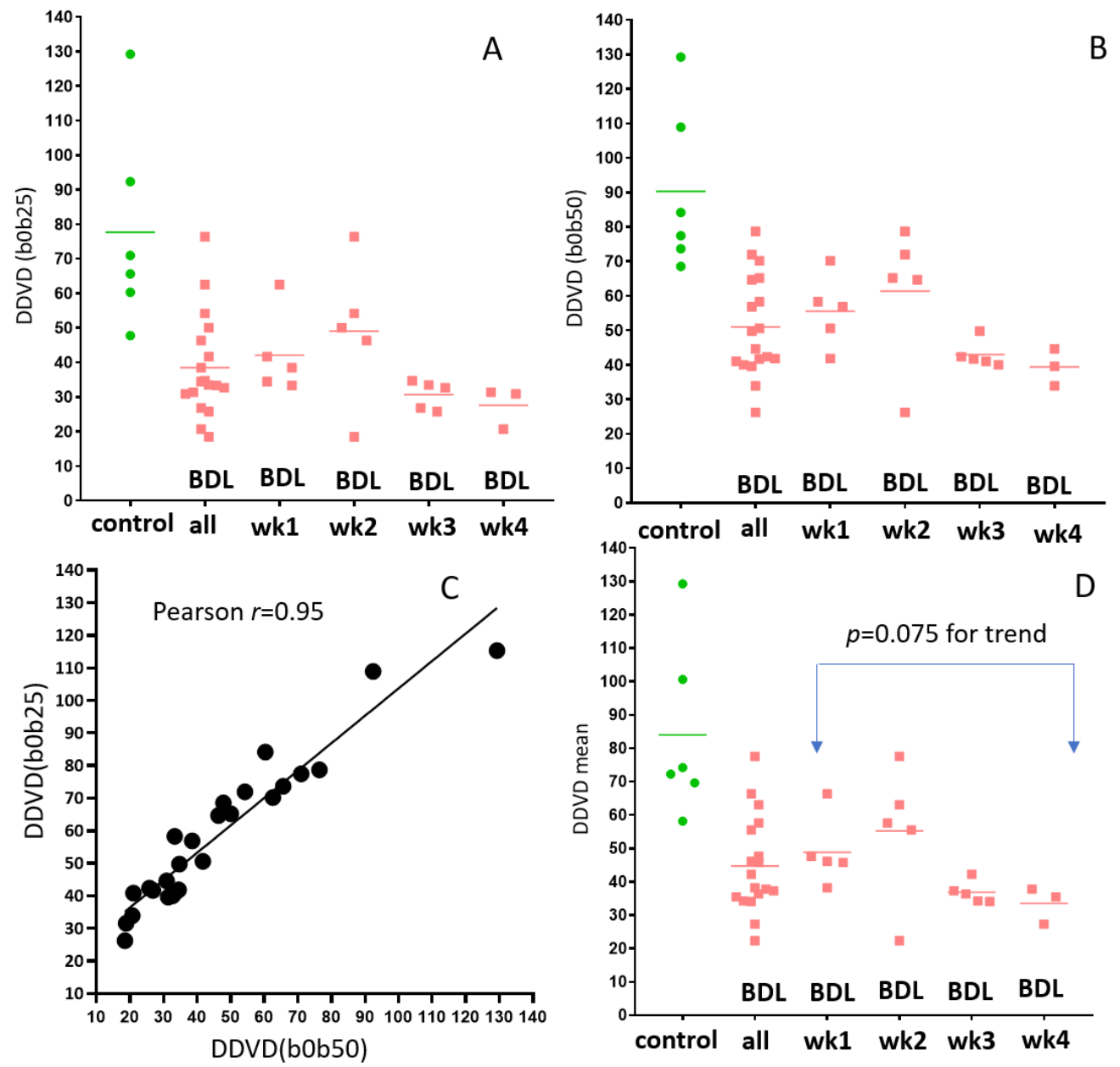

Fig-3. DDVD measures of control rats and rats with different time points post-BDL (biliary duct ligation). $A$ shows the results of $D D V D_{(b 0 b 25)}$. B shows the results of $D D V D_{(b 0 b 50)}$. $C$ shows the correlation between $\operatorname{DDVD}_{(\mathrm{b} 0 \mathrm{~b} 25)}$ and $\operatorname{DDVD}_{(\mathrm{b} 0 \mathrm{~b} 50)}$. $\mathrm{D}$ shows the results of $\mathrm{DDVD}_{\text {mean }}$. All: all rats with BDL $(n=18)$. 
PF alone could not totally separate healthy livers and all fibrotic livers ( $n=30, B D L$ rats and BDL rats with recanalization) (Fig-4A). According to equation-5, a 3-D space was constructed initially with PF, $D_{\text {slow, }}$ and $D_{\text {fast }}(F i g-4 B)$, and then additional two 3-D spaces was constructed by replacing $D_{\text {fast }}$ by $D D V D_{\text {mean }}$, or by replacing $D_{\text {slow }}$ by $D D V D_{\text {mean }}[2]$. All 3-D spaces totally separated healthy livers and all fibrotic livers. For these three 3-D spaces, the mean relative distances between healthy liver cluster and fibrotic liver cluster were respectively 0.331 for PF, $D_{\text {slow, }}$ and $D_{\text {fast }}, 0.381$ for $P F, D D V D_{\text {mean }}$, and $D_{\text {fast }}$, and 0.384 for $P F, D_{\text {slow, }}$ and $D D V D_{\text {mean }}$. With the assumption that bigger distance suggests potential better separation of non-fibrotic liver and fibrotic livers, the best separation of healthy liver and fibrotic livers was achieved with a combination of PF, $\mathrm{D}_{\text {slow, }}$ and DDVD mean $($ Fig-4C).

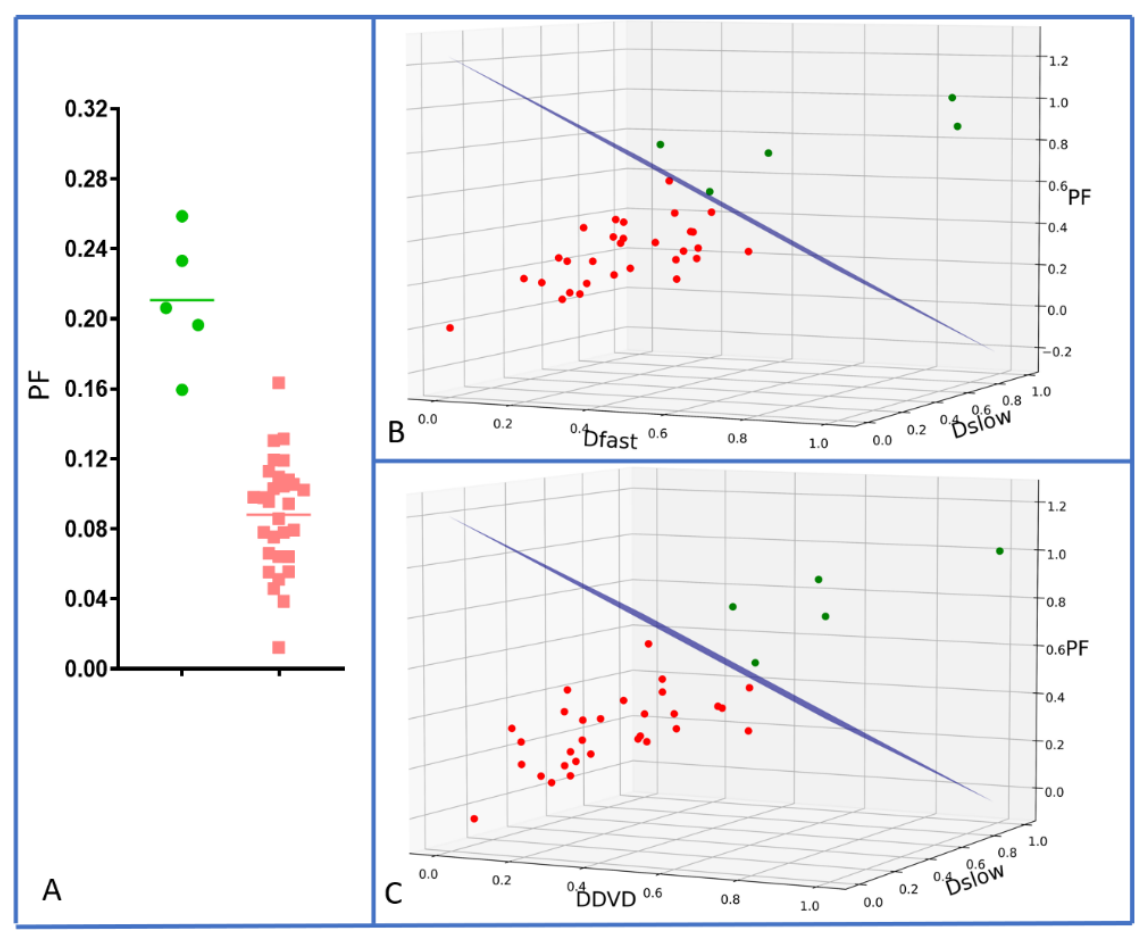

Fig-4. Separation of healthy livers $(n=5)$ and all fibrotic livers $(n=30, B D L$ rats and BDL+ recanalization rats) by diffusion MRI metrics. A: PF alone cannot totally separate healthy livers and all fibrotic livers. $B$ : a combination of PF, $D_{\text {slow, }}$ and $D_{\text {fast }}$ totally separates healthy livers and all fibrotic livers. C: compared with (B), a combination of PF, $D_{\text {slow, }}$ and $D D V D_{\text {mean }}$ even better separates healthy livers and all fibrotic livers. 


\section{Discussion}

In this study, a BDL model and a BDL-recanalization model were applied, and liver fibrosis of varying severity was included as expected (table-1). A longer duration of BDL was associated with a higher mean liver fibrosis score. The BDL-recanalization model applied in this study has been described by dos Santos et al [6]. This type of operation is associated with complications. The propulsive power of intestine wall can add to the biliary duct pressure, leading to bowelbiliary reflux. Free entering of the enteric content to the biliary duct may induce episodes of mechanical obstruction, leading to cholestasis and cholangitis. These were confirmed in this study by the continued existence of dilated biliary duct anatomically. The mean of liver fibrosis score of the re-cannulation group was higher than the week-1 group (2.42 vs 1.5), suggesting overall progress of liver fibrosis following the re-recanalization surgery.

The liver fibrosis caused with biliary duct blockage confirms what we have observed in our human patient studies that a combination of $P F, D_{\text {slow, }}$ and $D_{\text {fast }}$ allows a total separation of all healthy livers and fibrotic livers (Fig-4B). Thus, this study further supports our previous results that diffusion MRI can be used to detect even stage-1 fibrosis (four stage-1 fibrosis livers in this study) $[4,5]$. This study also confirmed that the integration of DDVD into the IVIM dataset improves the total separation of healthy livers and livers.

Declaration of Conflicting Interests: The authors declared no potential conflicts of interest with respect to the research, authorship, and/or publication of this article.

Funding: This work was supported by grants from the Shenzhen Basic Research Plan Fund Project (No. JCYJ20180228164641207) and the Cultivating Fund Project of Shenzhen People's Hospital (No. SYKYPY201933), and partially supported by a grant from the Research Grants Council of Hong Kong SAR (Project Nos. 2141061). 


\section{References}

1. Wang YXJ. Living tissue intravoxel incoherent motion (IVIM) diffusion MR analysis without b=0 image: an example for liver fibrosis evaluation. Quant Imaging Med Surg 2019,9,127-133.

2. Xiao BH, Huang H, Wang LF, Qiu SW, Guo SW, Wang YXJ. Diffusion MRI Derived per Area Vessel Density as a Surrogate Biomarker for Detecting Viral Hepatitis B-Induced Liver Fibrosis: A Proof-of-Concept Study. SLAS Technol. 2020;25:474-483.

3. Huang H, Zheng CJ, Wang LF, Che-Nordin N, Wáng YX. Age and gender dependence of liver diffusion parameters and the possibility that intravoxel incoherent motion modeling of the perfusion component is constrained by the diffusion component. NMR Biomed. 2021;34:e4449.

4. Wang YXJ, Wang X, Wu P, Wang Y, Chen W, Chen H, Li J. Topics on quantitative liver magnetic resonance imaging. Quant Imaging Med Surg 2019;9:1840-1890

5. Wang YX, Huang H, Zheng CJ, Xiao BH, Chevallier O, Wang W. Diffusion-weighted MRI of the liver: Challenges and some solutions for the quantification of apparent diffusion coefficient and intravoxel incoherent motion. Am J Nucl Med Mol Imaging 2021 ajnmmi0129123

6. dos Santos JS, Kemp R, de Andrade MF, Neder L. Influence of biliary anastomosis on recovery from secondary biliary cirrhosis. Eur J Gastroenterol Hepatol. 2012;24:1039-50.

7. Chevallier O, Zhou N, He J, Loffroy R, Wang YXJ. Removal of evidential motion-contaminated and poorly fitted image data improves IVIM diffusion MRI parameter scan-rescan reproducibility. Acta Radiol. 2018;59:1157-1167.

8. Wang YX, Li YT, Chevallier O, Huang H, Leung JCS, Chen W, Lu PX. Dependence of intravoxel incoherent motion diffusion MR threshold $b$-value selection for separating perfusion and diffusion compartments and liver fibrosis diagnostic performance. Acta Radiol. 2019;60:3-12. 